\title{
EINE ARMSCHUTZSPIRALE VOM TYP SALGÓTARJÁN MIT TEXTILRESTEN VON „STAMPFEN“ (STUPAVA)
}

\author{
VALENTINA GRAF-KARINA GRÖMER - GERHARD TR N A
}

\begin{abstract}
A B ronze Arm Protection Spiral of the Salgótarján Type with Remains of Textile from 'Stampfen' (Stupava). An old find of a bronze arm protection spiral of the Salgótarján type from the former 'Stampfen' in today's south-west Slovakia is presented. This typical product of the Carpathian Piliny culture (Slovakia and Hungary) dates from the late Middle Bronze Age to the late Bronze Age (BC-BD and HA1). Mineralized textile residues are a special feature at some points of the spiral. Here it is made clear that even old finds can still bring surprises when viewed closely.
\end{abstract}

Keywords: Western Slovakia, Late Bronze Age, bronze arm protector of the Salgótarján type, textiles, analysis.

In der Lehr- und Studiensammlung des Instituts für Urgeschichte und Historische Archäologie (vormals Institut für Ur- und Frühgeschichte) der Universität Wien befindet sich eine Bronzespirale vom Typ Salgótarján. Ihre Herkunft ist am scheibenförmigen Mittelstück mit „Stampfen (Ungarn)“ und der heutigen Inventarnummer der Sammlung ersichtlich. Weiters ist eine beschädigte alte Etikette mit den Zahlen „247“ (?) aufgeklebt. Stampfen ist der deutsche Name des heutigen Stupava (ungarisch Stomfa) in der Südwestslowakei an den westlichen Ausläufern der Kleinen Karpaten nördlich von Bratislava. Es ist somit anzunehmen, dass die Spirale im ehemaligen Gebiet des Königreichs Ungarn (damals Komitat Pozsony) im 19. Jahrhundert gefunden wurde. Weitere Funde aus "Stomfa" gelangten damals in das Ungarische Nationalmuseum Budapest (Bartík/Farkaš/Jelínek 2019, 38), haben aber zeitlich und auch fundumständehalber keinen Bezug zu unserer Armschutzspirale.

Die Spirale kam 1921 mit dem Ankauf der Sammlung Richard Böhmker (bzw. Richard Boehmker 1870-1954) ohne nähere Angaben an das „Urgeschichtliche Institut" (Felgenhauer 1965, 23 f.; www.uhasammlung.univie.ac.at/sammlung/ biographien/boehmker).

Die bronzene Armschutzspirale (Abb. 1; 2), ${ }_{1}^{1}$ interpretativ auch als Handschutzspirale bezeichnet (B. Hellebrandt 1996a, 30; 1996b, 186), besteht aus einem vierkantigen bzw. rhombischen Stabkörper mit einer linkslaufenden, neunfach gewundenen flachen Scheibe, zu der rechtwinkelig die rundstabige Armwindung mit einer flach gehämmerten Öse endet. In der ersten Spiralwindung ist ein bewegliches, separat gegossenenes, scheibenförmiges Mittelstück mit Dorn und einer hinteren Verstrebung an einem Niet eingesetzt. Der Durchmesser der Dornscheibe beträgt 3,7-3,8 cm, die Dornhöhe 1,5 cm. Die hintere, rhombische Verstrebung ist 2,4 cm lang, $0,5 \mathrm{~cm}$ breit und bis zu $0,8 \mathrm{~cm}$ hoch und schließt mit einer kleinen dreieckigen Erhöhung (Niet), wohl dem Rest des einstigen Gusstrichters beim Guss des Mittelstückes ab (siehe G. Tarbay 2014, Abb. 4). Die Höhe des Mittelstücks beträgt $3,7 \mathrm{~cm}$.

Der Stabkörper ist bis zu seiner zweiten Windung bandförmig gearbeitet (Stärke 0,3-0,4 cm) und an der Vorderseite von der Dornscheibe (Mittelstück) bedeckt. Ab der zweiten Windung ist der Stab rhombisch, mit einer zunehmenden Breite von $0,6-0,8 \mathrm{~cm}$ und einer durchgehenden Dicke von $0,8 \mathrm{~cm}$ ausgeformt. Die zwei Flächen des gerade verlaufenden rhombischen Stabes links und rechts des Mittelgrats weisen fünf Paare gegenständiger Linienfelder aus neun bis zwölf parallelen, feinen Linien auf. Die korrespondierenden Flächen auf der Stabrückseite sind unverziert. Am Mittelgrad der Vorderseite sind vom unteren Stabteil bis zur Hälfte der äußersten Spiralwindung in regelmäßigen Abständen feine Einkerbungen angebracht. Die äußere Spiralwindung biegt im untersten Bereich aus der Scheibe nach rückwärts, um dann aufwärts gebogen vor der Spirale gerade zur Armwindung zu verlaufen. An dieser Stelle geht das Oberteil rechtwinkelig in den rundstabigen, einfach gewundenen Armteil (Stabdurchmesser $0,8 \mathrm{~cm})$ über. Dieser ist an seinem Ende flachgehämmert und zu einer Öse eingerollt (Höhe $0,5 \mathrm{~cm}$ ). Die gesamte Spirale misst 23,5 cm, der Spiralscheibendurchmesser $15 \mathrm{~cm}$, der innere elliptische Armringdurchmesser 12,5 × $8 \mathrm{~cm}$. Das Gewicht beträgt $1080 \mathrm{~g}$.

Die Spirale ist ein in einem Stück (Stab) gegossenes und überschmiedetes, vollständig erhaltenes Fertigprodukt mit einem separat gegossenen

\footnotetext{
1 Metallographische Untersuchungen liegen nicht vor.
} 


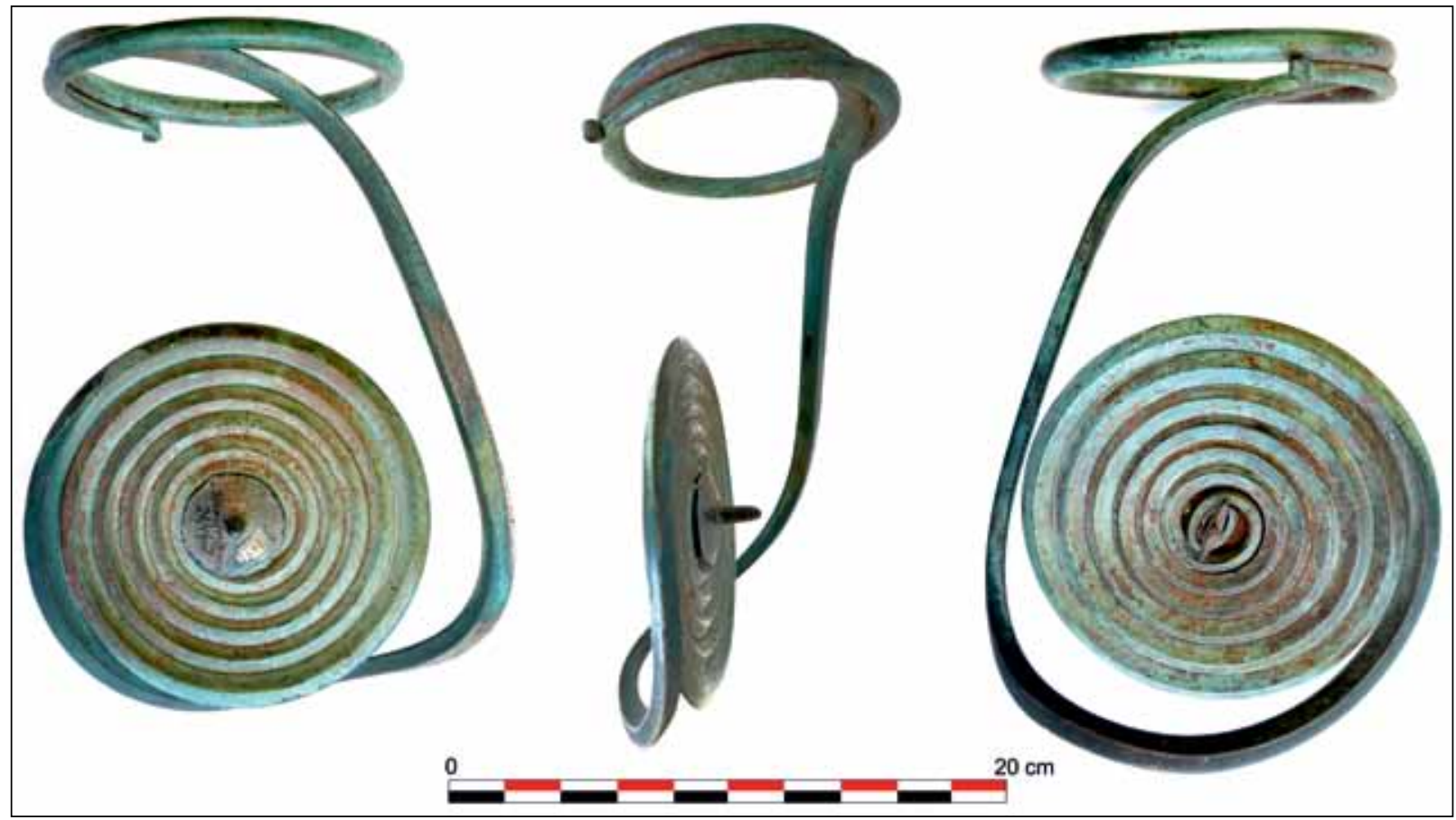

Abb. 1. Armschutzspirale Typ Salgótarján von „Stampfen (Ungarn)“. Institut für Urgeschichte und Historische Archäologie der Universität Wien, Inv.-Nr. 26463 (Foto G. Trnka).

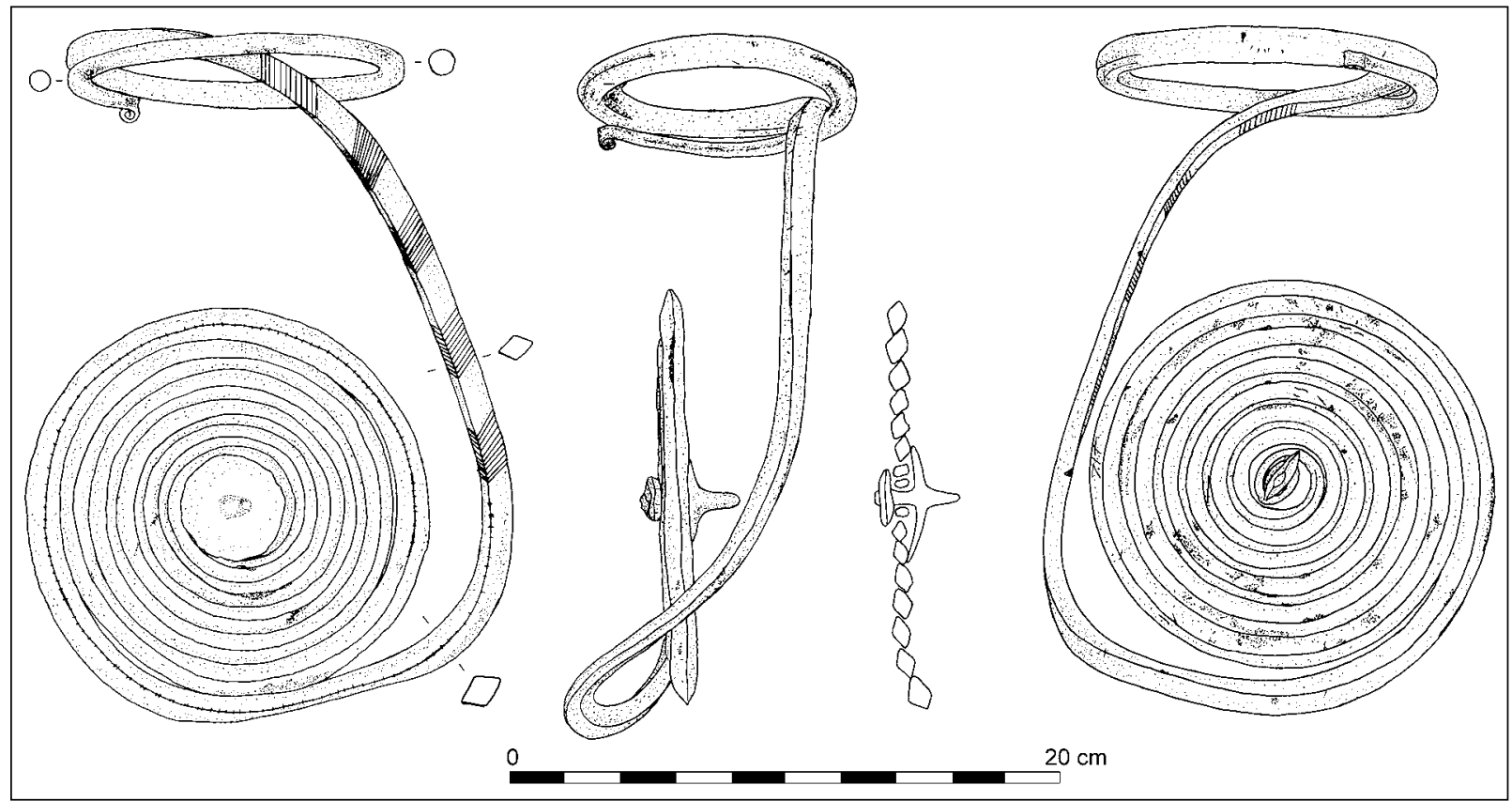

Abb. 2. Armschutzspirale Typ Salgótarján von „Stampfen (Ungarn)“. Institut für Urgeschichte und Historische Archäologie der Universität Wien, Inv.-Nr. 26463 (Grafik V. Graf).

Mittelstück. Die Oberfläche weist geringfügige Beschädigungen auf. Die unbeschädigten Stellen sind (dunkel)grün patiniert. Stellenweise ist die Patina abgeplatzt und zeigt ab der dritten Spiralwindung zunehmend Korrosionsstellen. Alte
Schlagspuren können im Bereich des Ansatzes der Armwindung, am Mittelstab und am Grat der zwei äußeren Windungen festgestellt werden. Weiters sind auch jüngere Beschädigungen zu sehen, wie blanke Stellen zeigen. 


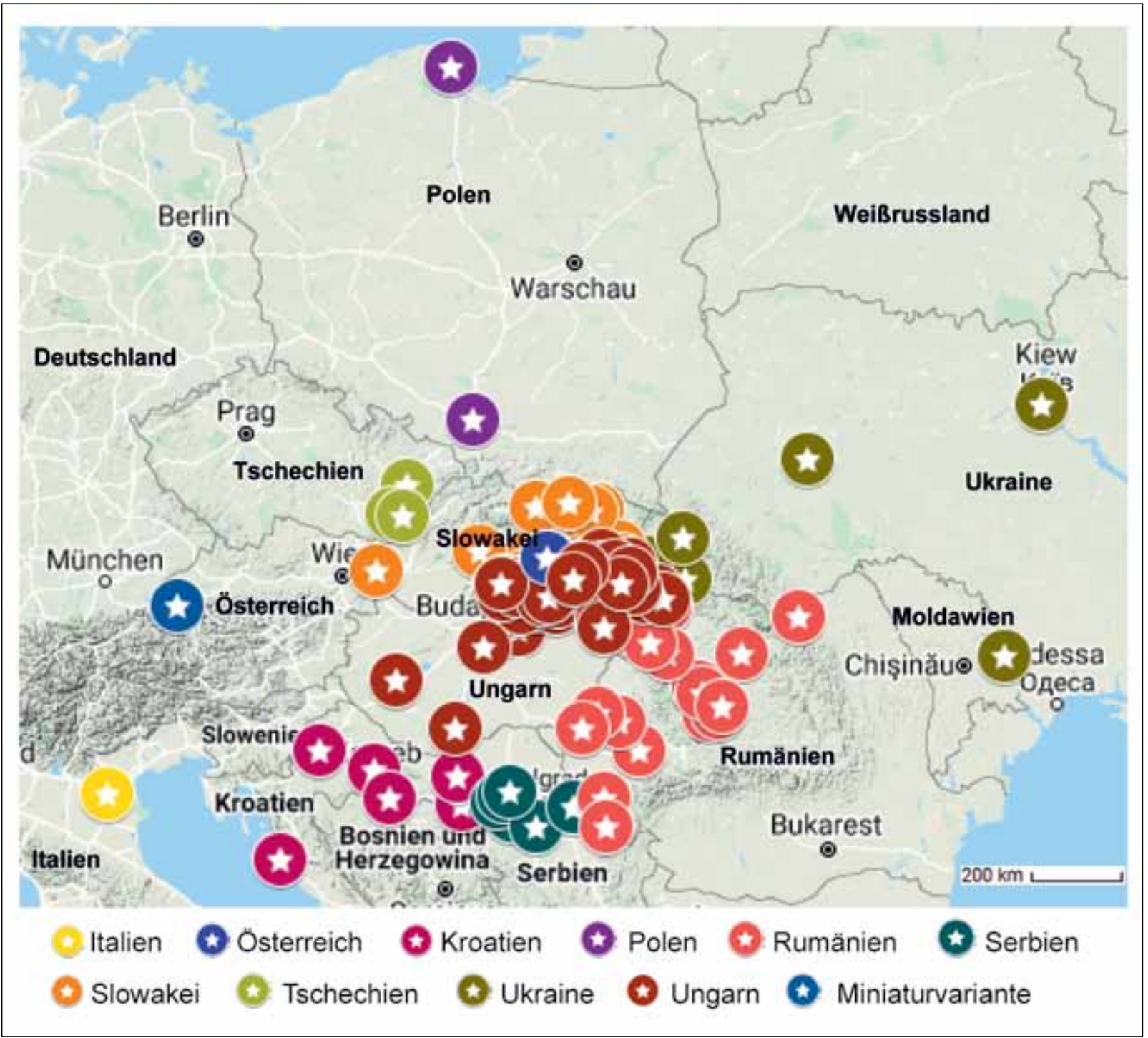

Abb. 3. Verbreitung der Armschutzspiralen vom Typ Salgótarján (Grafik V. Graf, hergestellt mittels GoogleMaps).

Die massiven bronzenen Armschutzspiralen vom Typ Salgótarján waren in der Bronzezeitforschung des Karpatenraumes schon immer Gegenstand von Überlegungen zur Zeitstellung wie auch zu ihrer nach wie vor nicht wirklich gelösten Verwendung bzw. Tragweise. Deren Häufung im Verband von spätmittel- bis spätbronzezeitlichen Bronzedepots in den Bereichen der Kupfererzreviere Nordungarns und der Süd(ost)slowakei (Abb. 3; siehe auch G. Tarbay 2014, Abb. 16) ist auf die entsprechend zur Verfügung stehenden Rohstoffquellen zurückzuführen (Kemenczei 1984, 25). Oft sind diese Spiralen in Depots paarweise links- und rechtsläufige Exemplare (B. Hellebrandt 1996a, 30, Abb. 12; 19; 1996b, 186, Abb. 10: 1; 11; Trnka 2015, Abb. 6; 7), sie treten aber auch einzeln in diesen auf.

Der Spiraltyp Salgótarján gehört grundsätzlich der ausgehenden Mittelbronzezeit (Stufe BC), zu- meist der Stufe BD und der Stufe HA1 an und ist eines der Kennzeichen der karpatischen Piliny Kultur (zuletzt G. Tarbay 2014, 84, 86, Abb. 13; Trnka 2015, 469 f.), wenngleich sie darüber hinaus ein weitläufiges Verbreitungsgebiet aufweisen (Abb. 3).

\section{DIE TEXTILEN ÜBERRESTE AUF DER SPIRALE VON STAMPFEN/STUPAVA (Karina Grömer)}

Mit freiem Auge nicht wahrnehmbar, sind an einigen Stellen der Rückseite der Spiralscheibe Spuren mineralisierter Textilreste im Zentrum erhalten geblieben (Abb. 4). Textile Überreste aus der Mittel- und Spätbronzezeit in Mitteleuropa sind relativ selten, können jedoch in besonderen Fällen an Metallelementen in Bodenlagerung erhalten 


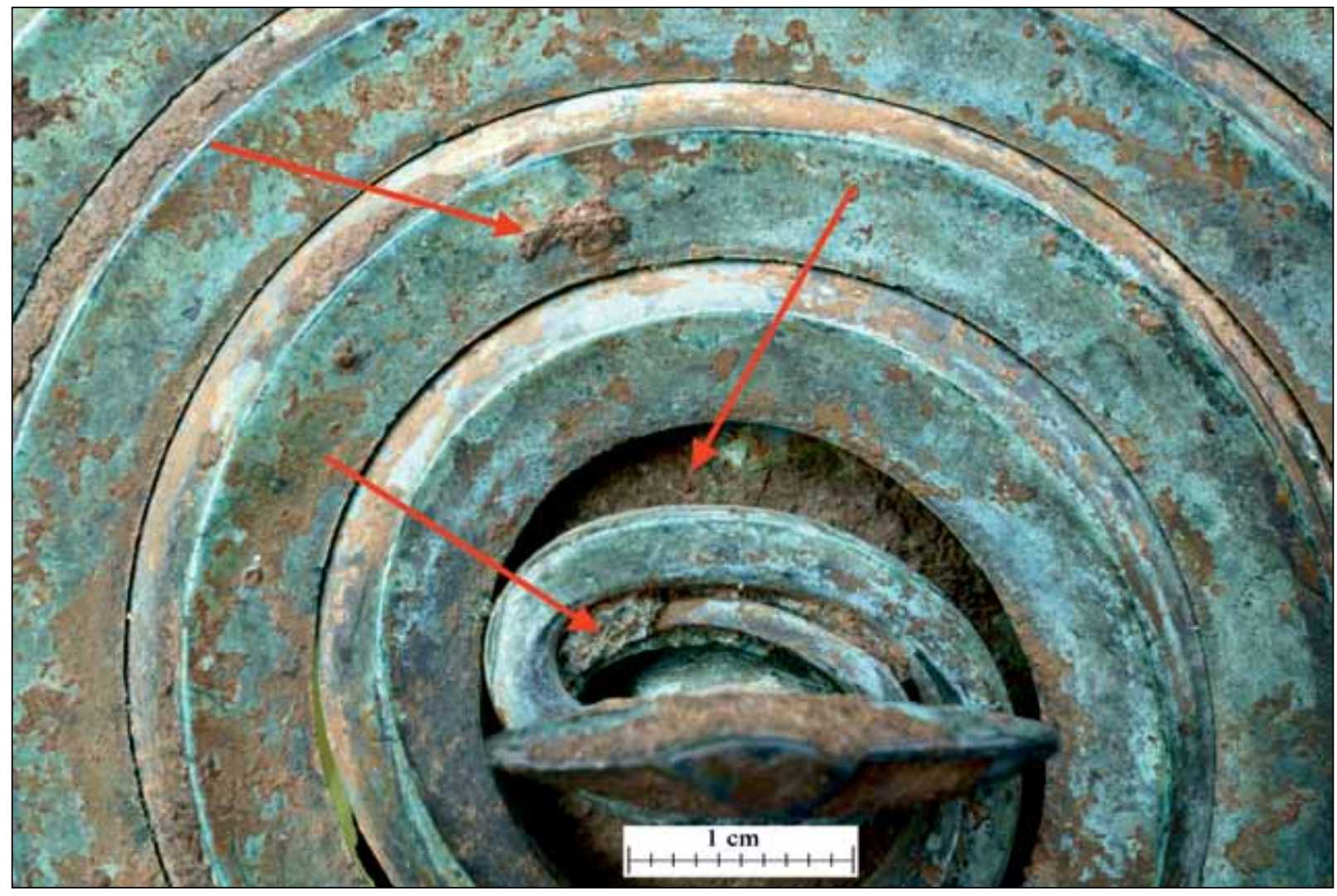

Abb. 4. Armschutzspirale Typ Salgótarján von „Stampfen (Ungarn)“ mit mineralisierten Textilresten (Detail). Institut für Urgeschichte und Historische Archäologie der Universität Wien, Inv.-Nr. 26463 (Foto G. Trnka).

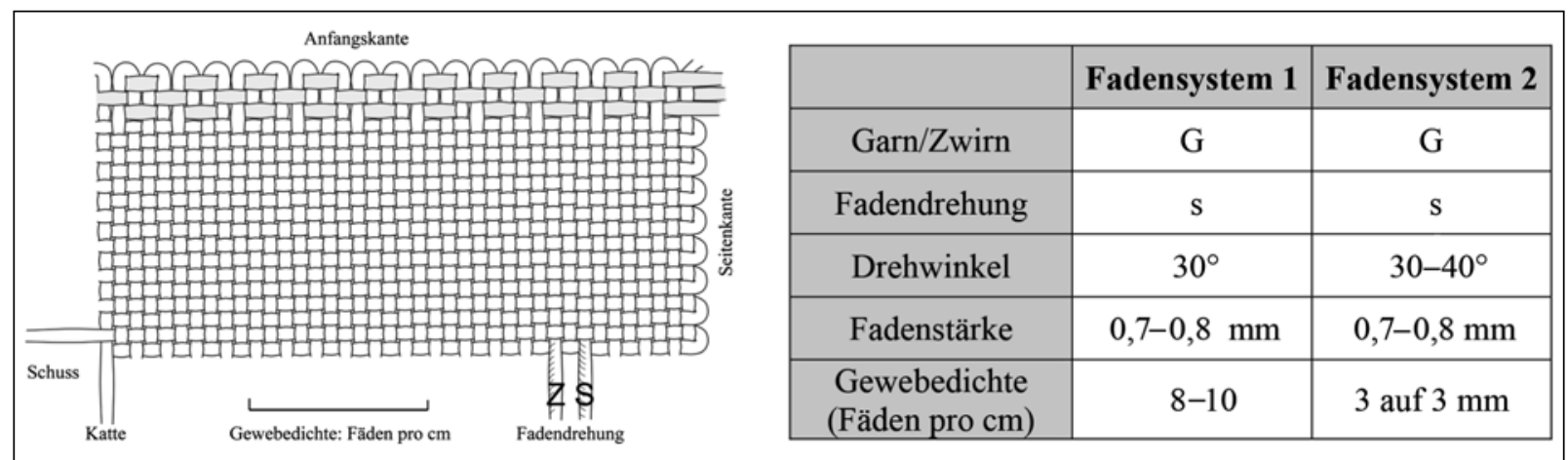

Abb. 5. Technische Daten zum Textilrest an der Armschutzspirale von „Stampfen (Ungarn)“ und Schema zu den gewebetechnischen Merkmalen (Grafik K. Grömer).

bleiben, wenn durch die Einflüsse der Lagerungsumgebung bei Metallen eine chemische Reaktion ausgelöst wird (Mitschke 2001, 29). Zunächst entsteht Korrosion (Oxidation), die stoffliche Veränderung metallischer Oberflächen. Wenn bei diesem Vorgang organische Materialien in Berührung mit Metalloberflächen kommen, werden die organischen Stoffe (Leder, Textil, Pflanzen) in anorganische umgewandelt (Mineralisierung).
Auf der Bronzespirale haben sich mineralisierte Textilreste an drei Stellen erhalten (Abb. 4). Direkt beim Niet im Zentrum der Spirale findet sich ein wenige Quadratmillimeter großer, grünlich mineralisierter Textilrest sowie auf der zweiten Windung an der Oberseite der Spirale. Aber auch an vereinzelten Stellen der Rückseite der Spiralplatte haben sich derartige Textilreste erhalten. Die Interpretation dieser Reste ist schwierig. Im Bereich des Nietes 


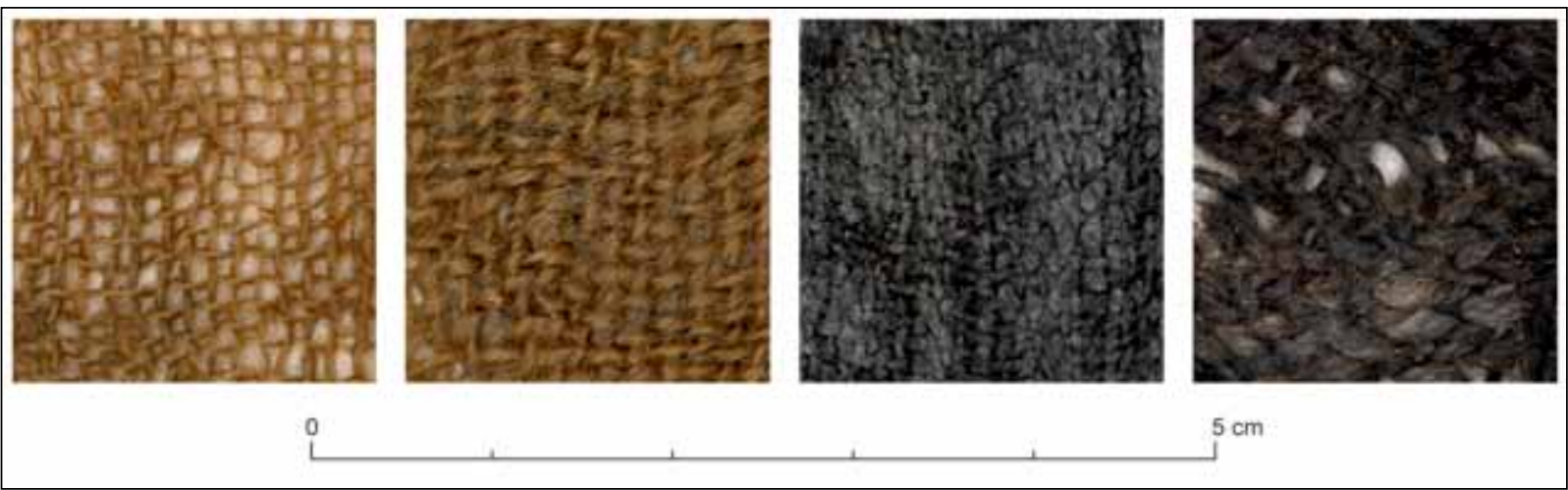

Abb. 6. Organisch erhaltene bronzezeitliche Gewebe aus dem Salzbergwerk Hallstatt, ca. 1400-1000 v. Chr. in Leinwandbindung (@ Naturhistorisches Museum Wien).

wurde offenbar ein Textil durch das Verbinden von Armschutzspirale und Unterlage zwischen dem Niet und der Öffnung im Zentrum der Spirale eingeklemmt.

Die Analyse des Textilrestes erfolgte sowohl auf makroskopischer wie mikroskopischer Ebene (Grömer 2014, 9-16). Die Gewebestruktur und -qualität wurde mit einem Digitalmikroskop (DinoLite Digital Microscope) dokumentiert, wobei mit Vergrößerungen zwischen 30- und 250-fach gearbeitet werden kann. Die zugehörige Software ermöglicht es, Fadenstärken und Drehwinkel (Stärke der Fadendrehung) zu ermitteln. Die Fadenstärken, der Verlauf der Kett- und Schussfäden und auch feinste Gewebestrukturen lassen sich so erfassen. Technische Daten, die von einem Gewebe aufgenommen werden können, sind Bindungsart, Fadendichte, Fadenstärke, Zwirn oder Garn, S- oder Z-Drehung, verschiedene Webfehler, diverse Nähte und Säume sowie gegebenenfalls Verzierungen (Abb. 5).

Im konkreten Fall handelt es sich beim an der Armschutzspirale von "Stampfen" erkennbaren Textilrest um ein leinwandbindiges Textil, das im Vergleich mit anderen bronzezeitlichen Geweben mittelfein mit einer Fadenstärke von 0,7-0,8 mm und in einer erkennbaren Gewebedichte um 8-10 Fäden pro cm ist (Abb. 5). Das Rohmaterial, aus dem das Gewebe gefertigt wurde, kann aufgrund des schlechten Erhaltungszustandes nicht bestimmt werden. Auch ist die Ausrichtung des Gewebes, in welche Richtung Kette und Schuss liegt, nicht $\mathrm{zu}$ identifizieren, weshalb hier allgemein bei der Beschreibung des Gewebes von Fadensystem 1 und Fadensystem 2 gesprochen wird.

\section{VERGLEICHE ZU ANDEREN BRONZEZEITLICHEN GEWEBEN}

Bronzezeitliche Textilfunde in Zentraleuropa sind an Metallobjekten aus Gräbern bekannt, selten von Siedlungskontexten, etwa als Eindrücke auf Keramik (Grömer 2009). Von den bronzezeitlichen Kupferbergwerken Mitterberg/Hochkönig und Radfeld (Grömer u. a. 2017) sind Stoffreste in Feuchterhaltung aus dem Areal der Aufbereitungsanlagen organisch erhalten. Besonders herausragend sind die Funde aus den Salzbergwerken in Hallstatt (Grömer u. a. 2013), die zahlenmäßig die größte Gruppe darstellen. Die ältesten Gewebe aus Hallstatt stammen von den bronzezeitlichen Fundpunkten der Nordgruppe und des Tuschwerkes (ca. 1400-1000 v. Chr.).

Der Textilrest fügt sich puncto Bindungsart (Leinwandbindung), Fadenstärke, Gewebedichte und bezüglich des verwendeten Fadenmaterials gut in das bekannte Bild bronzezeitlicher Gewebe ein (Abb. 6). 


\section{LITERATUR}

Bartík/Farkaš/Jelínek 2019 - J. Bartík/Z. Farkaš/P. Jelínek: Bronzehortfunde aus den Kleinen Karpaten und ihren Vorgebirgsgebieten. In: I. Bazovský/G. Březinová (ed.): Ĺudia a hory-archeologická perspektíva. Interakcie l’udských spoločenstiev horských a podhorských oblastí západného Slovenska. Zborník SNM. Archeológia. Supplementum 12. Bratislava 2019, 15-102.

B. Hellebrandt 1996a - M. B. Hellebrandt: A kurityáni bronzlelet. A Herman Ottó Múzeum Évkönyve 33-34, 1996, 5-31.

B. Hellebrandt $1996 b$ - M. B. Hellebrandt: Der Bronzefund von Kurityán. In: T. Kovács (Hrsg.): Studien zur Metallindustrie im Karpatenbecken und den benachbarten Regionen. Festschrift Amália Mozsolics zum 85. Geburtstag. Budapest 1996, 183-205.

Felgenhauer 1965 - F. Felgenhauer: Zur Geschichte des Faches "Urgeschichte" an der Universität Wien. Studien zur Geschichte der Universität Wien 3, 1965, 7-27.

Grömer 2009 - K. Grömer: Textilien der Bronzezeit in Mitteleuropa. Archaeologica Austriaca 90, 2006 (2009), 31-72.

Grömer 2014 - K. Grömer: Römische Textilien in Noricum und Westpannonien im Kontext der archäologischen Gewebefunde 2000 v. Chr. -500 n. Chr. in Österreich. Mit Beiträgen von A. Paetz gen. Schieck, E. Hölbling-Steigberger und K. Gostenčnik. Austria Antiqua 5. Graz 2014.

Grömer u. a. 2013 - K. Grömer/A. Kern/H. Reschreiter/ H. Rösel-Mautendorfer: Textiles from Hallstatt. Weaving
Culture in Bronze and Iron Age Salt Mines = Textilien aus Hallstatt. Gewebte Kultur aus dem bronze- und eisenzeitlichen Salzbergwerk. Archaeolingua 29. Budapest 2013.

Grömer u. a. 2017 - K. Grömer/G. Goldenberg/J. Banck-Burgess/M. Gleba Margarita/R. Hofmann-de Keijzer/ M. van Bommel/I. Joosten/M. Mehofer/K. Nicolussi/ U. Töchterle: Textilreste aus einem spätbronzezeitlichen Bergbaurevier bei Radfeld in Nordtirol - Sekundärnutzung von Stoffen zur Abdichtung. Prähistorische Zeitschrift 92, 2017, 322-341.

G. Tarbay 2014 - J. G. Tarbay: The Central-European 'spiral arm-guard'. Notes on the Bronze Age asymmetrical arm- and anklets. Communicationes Archaeologicae Hungariae 2014, 2014, 71-106.

Kemenczei 1984 - T. Kemenczei: Die Spätbronzezeit Nordostungarns. Archaeologia Hungarica 51. Budapest 1984.

Mitschke 2001 -S. Mitschke: Zur Erfassung und Auswertung archäologischer Textilien an korrodiertem Metall. Eine Studie $z u$ ausgewählten Funden aus dem Gräberfeld von Eltville, Rheingau-Taunus-Kreis (5.-8. Jh.n. Chr.). Vorgeschichtliches Seminar der Philipps-Universität Marburg. Kleine Schriften 51. Marburg 2001.

Trnka 2015 - G. Trnka: Ein spätbronzezeitlicher Bronzedepotfund von Bátka aus dem ehemaligen Nordungarn. In: I. Szathmári (Hrsg.): An der Grenze der Bronze- und Eisenzeit. Festschrift für Tibor Kemenczei zum 75. Geburtstag. Budapest 2015, 459-476.

Manuskript angenommen am 20. 8. 2020

Abstract translated by Gerhard Trnka

Súhrn preložila Anita Kozubová

Valentina Graf

Andreas-Urteil-Weg 1/51/10

A - 1220 Wien

graf.valentina@gmail.com

Priv.-Doz. Mag. Dr. Karina Grömer

Prähistorische Abteilung

Naturhistorisches Museum Wien

Burgring 7

A - 1014 Wien

karina.groemer@nhm-wien.ac.at

ao. Univ.-Prof. Dr. Gerhard Trnka

Institut für Urgeschichte und Historische Archäologie Universität Wien

Franz-Klein-Gasse 1

A - 1190 Wien

gerhard.trnka@univie.ac.at 


\title{
Šalgotariánsky kruh so zvyškami textilu zo „Stampfenu“ (Stupava)
}

\author{
Valentina Graf - Karina Grömer - Gerhard Trnka
}

\section{SÚHRN}

Príspevok je venovaný nálezu bronzového šalgotariánskeho kruhu z bývalého „Stampfenu“ na území dnešného juhozápadného Slovenska, ktorý je súčastou študijnej zbierky Ústavu pravekej a historickej archeológie Viedenskej univerzity a bol nájdený pravdepodobne v 19. storočí. Tento typický výrobok pilinskej kultúry (Slovensko a Mad’arsko) je datovaný do záveru strednej a do mladšej doby bronzovej (stupne BC-BD a HA1). Jeho zvláštnostou sú mineralizované textilné zvyšky zachované na troch miestach špirály. Na základe makro- a mikroskopickej analýzy ide o zvyšky stredne jemnej plátenej tkaniny. Surovinu, z ktorej bola látka vyrobená, nie je možné určit z dôvodu zlého stavu konzervácie predmetu. Nálezy textilu z doby bronzovej sú v strednej Európe doložené nielen na kovových predmetoch $\mathrm{z}$ hrobov, ale zriedkavo aj zo sídliskových kontextov, napríklad ako odtlačky na keramike (Grömer 2009). Pokial ide o typ väzby (hladká väzba), hrúbku nití a použitý materiál pri ich výrobe ako aj o hustotu tkaniny, zvyšky textilu zo Stampfenu/Stupavy zapadajú do známeho obrazu o tkaninách $\mathrm{z}$ doby bronzovej. Predmetný masívny kruh je zaujímavým príkladom toho, že aj staré nálezy môžu pri detailnej analýze priniest’ nové vedecké prekvapenia. 
